\title{
Pulmonary Vein, Dorsal Atrial Wall and Atrial Septum Abnormalities in Podoplanin Knockout Mice With Disturbed Posterior Heart Field Contribution
}

\author{
YVONNE L. DOUGLAS, EDRIS A. F. MAHTAB, MONIQUE R. M. JONGBLOED, PAVEL UHRIN, JAN ZAUJEC, \\ BERND R. BINDER, MARTIN J. SCHALIJ, ROBERT E. POELMANN, MARCO C. DERUITER, \\ AND ADRIANA C. GITTENBERGER-DE GROOT
}

\begin{abstract}
Department of Cardio-thoracic Surgery [Y.L.D.], University Medical Center Groningen, Groningen 9700 RB, The Netherlands; Department of Anatomy and Embryology [Y.L.D., E.A.F.M., M.R.M.J., R.E.P., M.C.D., A.C.G.G.], Department of Cardiology [M.J.S.], Leiden University Medical Center, Leiden 2300 RC, The Netherlands; Department of Vascular Biology and Thrombosis Research [P.U., J.Z., B.R.B.], Center for Biomolecular Medicine and Pharmacology, Medical University of Vienna, Vienna A-1235, Austria
\end{abstract}

\begin{abstract}
The developing sinus venosus myocardium, derived from the posterior heart field, contributes to the atrial septum, the posterior atrial wall, the sino-atrial node, and myocardium lining the pulmonary and cardinal veins, all expressing podoplanin, a coelomic and myocardial marker. We compared development and differentiation of the myocardium and vascular wall of the pulmonary veins (PV), left atrial dorsal wall, and atrial septum in wild type with podoplanin knockout mouse embryos (E10.5-E18.5) by 3D reconstruction and immunohistochemistry. Expression of Nkx2.5 in the pulmonary venous myocardium changes from mosaic to positive during development pointing out a high proliferative rate compared with Nkx2.5 negative myocardium of the sino-atrial node and cardinal veins. In mutants, myocardium of the PVs, dorsal atrial wall and atrial septum was hypoplastic. The atrial septum and right-sided wall of the PV almost lacked interposed mesenchyme. Extension of smooth muscle cells into the left atrial body was diminished. We conclude that myocardium of the PVs, dorsal atrial wall, and atrial septum, as well as the smooth muscle cells, are derived from the posterior heart field regulated by podoplanin. (Pediatr Res 65: 27-32, 2009)
\end{abstract}

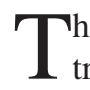
he developmental origin and molecular mechanisms controlling the formation of the myocardium of the pulmonary veins (PV) and the smooth muscle cells (SMCs) of the PV media are a matter of debate. The PV myocardial sleeve develops by either migration of myocardial cells from the left atrium (LA) (1) or by recruitment of extracardiac mesenchymal cells, which differentiate into myocardial cells (2). Based on expression patterns of $N k x 2.5$, Islet1, and Pitx $2 c$ the LA and PV myocardium was suggested to be formed by the addition of myocardial cells from the second heart field at the venous pole (3).

The second heart field, part of the splanchnic mesoderm, is involved in the addition of (myocardial) cells to the primary heart tube at both the arterial (the secondary and anterior heart field) and the venous pole (the posterior heart field) (4). Islet1, a marker of undifferentiated cardiac progenitor cells, is ex-

Received April 25, 2008; accepted August 13, 2008.

Correspondence: Adriana C. Gittenberger-de Groot, PhD, Department of Anatomy and Embryology, Leiden University Medical Center, P.O. Box 9600, Postal Zone S-1-P, 2300 RC Leiden, The Netherlands; e-mail: acgitten@lumc.nl

Y.L.D. and E.A.F.M. contributed equally to this study. pressed throughout the second heart field (5). Myocardial cells can differentiate from second heart field derived mesenchymal cells, and SMCs may also differentiate from mesenchyme (6).

The transmembrane glycoprotein podoplanin, a coelomic and myocardial marker expressed in the posterior heart field (7), has gained interest for its role in epithelial-tomesenchymal transformation (EMT) and in formation of the myocardium and coronary SMCs at the venous pole of the heart (8). It is found in the proepicardial organ, epicardium, and sinus venosus myocardium including the sino-atrial node, the PVs and cardinal veins, the dorsal atrial wall and the base of the atrial septum extending into the developing atrial and ventricular cardiac conduction system. Podoplanin promotes EMT by binding ezrin, radixin, moesin (ERM) proteins that activate RhoA, and by down-regulation of the cell-to-cell adhesion molecule E-cadherin (9). Lack of podoplanin leads to altered EMT and to abnormal formation of sinus venosus myocardium and decreased numbers of SMCs of the coronary artery media (8). In extracardiac tissues, podoplanin functions as a possible signaling molecule and is involved in the development of osteoblasts (10), intestinal and alveolar epithelium (11), podocytes and mesothelium of the visceral peritoneum (12), and lymphatic endothelium (13). During cardiac development, podoplanin is expressed specifically in the second heart field-derived myocardium and mesenchyme at the venous pole of the heart $(7,8)$.

Our previous study in humans (14) demonstrated that during incorporation of the PVs in the LA body, the inner lining of the LA body presents vessel wall tissue. The outer layer of the LA is formed by myocardium covered by epicardium. The origin of the SMCs of the LA dorsal wall is still a topic of discussion.

In the present study, we have compared the morphology and development of the wall of the PVs, LA dorsal wall, and atrial septum between wild type and podoplanin knockout mouse embryos of embryonic stages (E) 10.5-18.5. We hypothesize that the posterior heart field is involved in the formation and

Abbreviations: EMT, epithelial-mesenchymal transformation; LA, left atrium; PV, pulmonary vein; SMCs, smooth muscle cells 
differentiation of both myocardial cells and SMCs of the PVs and LA dorsal wall regulated by podoplanin.

\section{MATERIALS AND METHODS}

Generation of the embryonic and neonatal mice. This study was performed at the Department of Anatomy and Embryology of the Leiden University Medical Center, and was approved by the Animal Research Committee. Podoplanin knockout mice were generated by homologous recombination in embryonic stem cells from the $129 \mathrm{~S} / \mathrm{v}$ mouse line, as reported previously (8).

General description. We investigated the morphology and development of the sinus venosus region of the heart, especially the PVs, LA dorsal wall, and atrial septum in 32 wild-type mouse embryos of E10.5 $(n=4)$, E11.5 $(n=3)$, $\mathrm{E} 12.5(n=4), \mathrm{E} 13.5(n=5), \mathrm{E} 14.5(n=4), \mathrm{E} 15.5(n=3), \mathrm{E} 16.5(n=3)$, E17.5 $(n=3)$, and E18.5 $(n=3)$, and compared these with 28 podoplanin knockout mouse embryos of E10.5 $(n=4), \mathrm{E} 11.5(n=5), \mathrm{E} 12.5(n=5)$, $\operatorname{E} 13.5(n=3), \operatorname{E} 14.5(n=4), \operatorname{E} 15.5(n=4)$, and E18.5 $(n=3)$.

Immunohistochemistry. Immunohistochemistry was performed with antibodies against alpha-smooth-muscle actin (1A4, 1:2000, Sigma Chemical Co. Aldrich, Product No.A2547, USA) to detect differentiated SMCs and developing myocardium, atrial myosin light chain 2 (MLC2a, 1/6000, kindly provided by S.W. Kubalak, Charleston, SC) specific for (atrial) myocardium, NK2 transcription factor related locus 5 (Nkx2.5, 1/4000, Santa Cruz Biotechnology, CA, SC-8697) as an early marker of undifferentiated cardiac progenitor cells, and podoplanin (clone 8.1.1., 1/500, Hybridomabank, IA) as a marker of the posterior heart field myocardium. Fixation, preparation, and staining procedures were completed according to standard protocols (8).

$3 D$ reconstructions. We made $3 \mathrm{D}$ reconstructions, as described previously (8), of the composition of PV, LA dorsal wall, and atrial septum of MLC-2a and 1A4 stained sections of podoplanin wild-type and knockout embryos (E15.5).

Morphometry. Sinus venosus myocardial volume estimation was performed of 12 wild-type mouse hearts of E11.5 $(n=3)$, E12.5 $(n=3)$, E15.5 $(n=3)$, and E18.5 $(n=3)$ and 12 podoplanin knockout hearts of E11.5 $(n=$ 3), E12.5 $(n=3), \mathrm{E} 15.5(n=4)$, and E18.5 $(n=2)$. Statistical analysis was performed with an independent sample $t$ test $(p<0.05)$ using SPSS 11.0 software (SPSS Inc, Chicago, IL), as described previously (8).

\section{RESULTS}

Podoplanin mutants demonstrate marked mesenchymal and myocardial abnormalities, including hypoplasia of the proepicardial organ, abnormal epicardium, hypoplastic chamber myocardium, atrial, ventricular and atrioventricular septal defects, and an abnormal coronary arterial vascular wall $(7,8)$. These abnormalities are related to diminished EMT, which was reported in a previous article (8). Later, the morphologic development of the wall of the PV, LA, and atrial septum will be described in subsequent stages. Abnormal PV connections were not observed. In Table 1 , sinus venosus myocardial volumes between wild-type and knockout mice are compared. Table 2 provides an overview of qualitative expression of myocardial and SMCs markers used.

E10.5. In the right part of the common atrium, the right and left venous valves delineated the ostia of the cardinal veins entering the sinus venosus. In both wild-type and knockout embryos, MLC2a was expressed in the sinus venosus and chamber myocardium (Fig. 1a,c-e). In the mesenchyme of the dorsal mesocardium in wild-type embryos, the lumen of the primitive PV was surrounded by a mainly left sided concentration of MLC2a positive myocardium that stained, however, less markedly for MLC2a compared with the common atrium (Fig. $1 a, d$ ). In knockouts, this myocardium was thinner with clearly diminished MLC2a expression in a larger part in the dorsal mesocardium and left part of the atrial dorsal wall (compare Fig. 1d with $e$ ). This diminished expression was seen in the region normally expressing podoplanin (Fig. $1 b$,

Table 1. Myocardial morphometry

\begin{tabular}{|c|c|c|c|c|c|}
\hline \multirow[b]{2}{*}{$\mathrm{E}$} & \multicolumn{2}{|l|}{ WT } & \multicolumn{2}{|c|}{ Podoplanin $^{-/-}$} & \multirow[b]{2}{*}{$t$-Test $p$ value } \\
\hline & Myocardial volume $\left(\mathrm{mm}^{3}\right)$ & SD & Myocardial volume $\left(\mathrm{mm}^{3}\right)$ & SD & \\
\hline 11.5 & $0.0094(n=3)$ & 0.0029 & $0.0050(n=3)$ & 0.0017 & 0.0457 \\
\hline 12.5 & $0.0120(n=3)$ & 0.0025 & $0.0071(n=3)$ & 0.0032 & 0.0348 \\
\hline 15.5 & $0.0424(n=3)$ & 0.0052 & $0.0300(n=4)$ & 0.0047 & 0.0108 \\
\hline 18.5 & $0.2871(n=3)$ & 0.0307 & $0.1688(n=2)$ & 0.0287 & 0.0115 \\
\hline
\end{tabular}

Sinus venosus myocardial volume estimation of 12 wild type (WT) and 12 podoplanin $^{-1-}$ mouse hearts. In all stages, mutants have a significant smaller myocardial volume $(p<0.05)$ compared with WT embryos.

E, embryonic day; SD, standard deviation.

Table 2. Expression of markers

\begin{tabular}{|c|c|c|c|c|c|c|c|c|c|}
\hline E & Markers & $\begin{array}{l}\text { PV } \\
\text { WT }\end{array}$ & $\begin{array}{c}\text { Wall } \\
\text { Mutant }\end{array}$ & $\begin{array}{l}\text { LA dorsal } \\
\text { WT }\end{array}$ & $\begin{array}{c}\text { Wall } \\
\text { Mutant }\end{array}$ & $\begin{array}{l}\text { Atrial } \\
\text { WT }\end{array}$ & $\begin{array}{l}\text { Septum } \\
\text { Mutant }\end{array}$ & $\begin{array}{l}\text { Media } \\
\text { WT }\end{array}$ & $\begin{array}{c}\text { PV } \\
\text { Mutant }\end{array}$ \\
\hline \multirow[t]{3}{*}{10.5} & MLC2a & ++ & + & ++ & + & \multicolumn{4}{|c|}{ Not developed yet } \\
\hline & Nkx2.5 & ++ & + & ++ & + & & & & \\
\hline & Actin & ++ & + & ++ & + & & & & \\
\hline \multirow[t]{3}{*}{12.5} & MLC2a & ++ & + & +++ & ++ & +++ & ++ & ++ & ++ \\
\hline & Nkx2.5 & ++ & + & ++ & ++ & ++ & ++ & ++ & ++ \\
\hline & Actin & + & + & + & + & + & + & - & - \\
\hline \multirow[t]{3}{*}{15.5} & MLC2a & +++ & +++ & +++ & +++ & +++ & +++ & + & + \\
\hline & Nkx2.5 & +++ & +++ & +++ & +++ & +++ & +++ & + & + \\
\hline & Actin & - & - & - & - & - & - & ++ & + \\
\hline \multirow[t]{3}{*}{18.5} & MLC2a & +++ & +++ & +++ & +++ & +++ & +++ & - & - \\
\hline & Nkx2.5 & +++ & +++ & +++ & +++ & +++ & +++ & - & - \\
\hline & Actin & - & - & - & - & - & - & +++ & + \\
\hline
\end{tabular}

A qualitative overview of the expression of markers at several locations in podoplanin WT and knockout (KO) embryos. At early stages, the expression of all markers is diminished in mutants compared to the WT embryos, whereas in advanced stages these differences are no longer apparent, indicating delayed differentiation in mutants.

LA, left atrium; PV, pulmonary vein; +++, strong; ++, medium; +, weak; -, absent expression. 


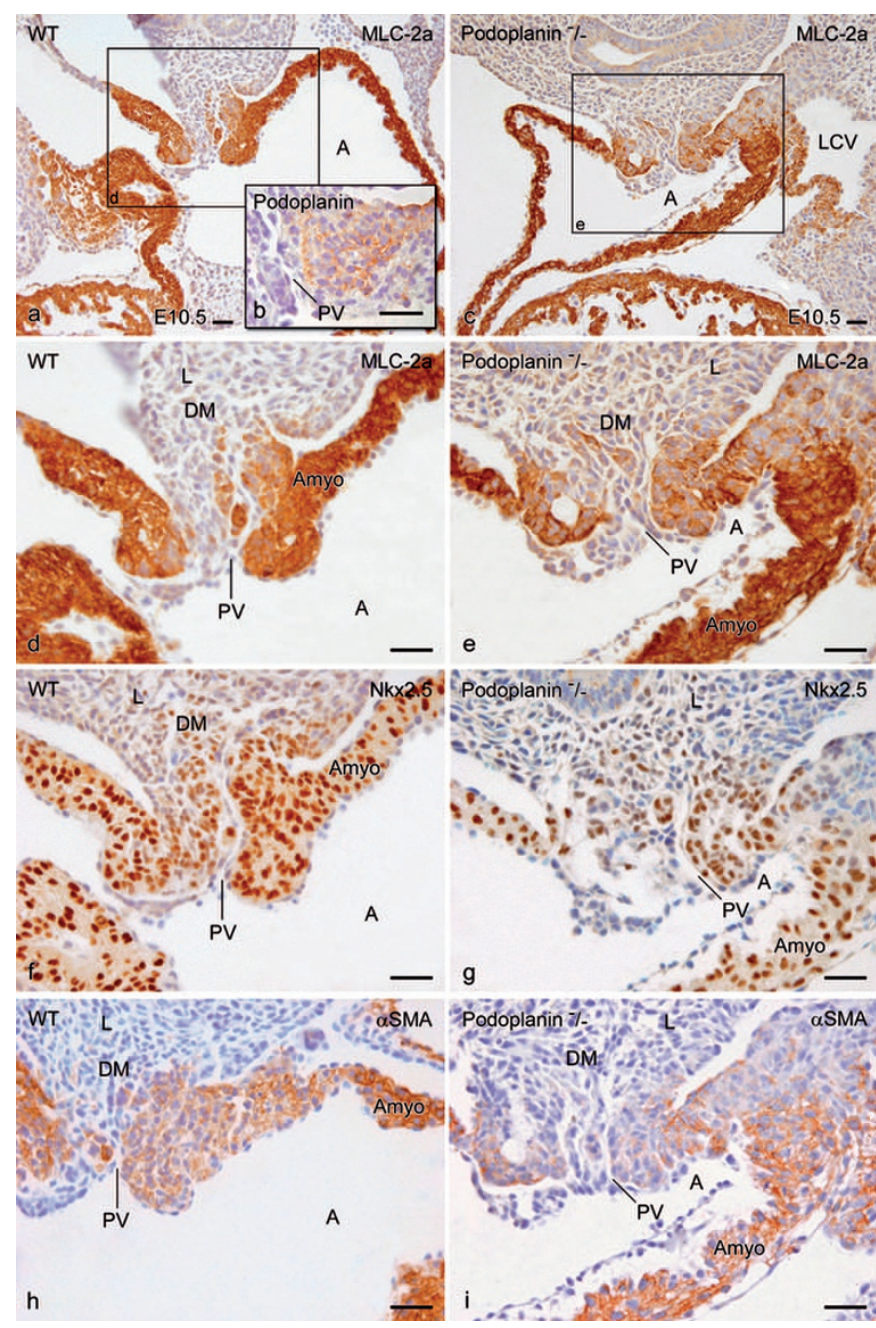

Figure 1. Transverse sections of podoplanin wild type (WT, $a, b, d, f, h)$ and knockout (Podoplanin-l-, $c, e, g, i$ ) mouse embryos of stage (E) 10.5 comparing the development of the pulmonary vein (PV). Boxed area in $a$ and $c$ are magnified in $d$ and $e$. MLC2a expression is seen around the primitive PV on the left side of the dorsal mesocardium (DM) $(a, d)$ where also podoplanin $(b)$ and mosaic Nkx2.5 expression $(f)$ is seen. Compared with the atrial myocardium (Amyo), the MLC2a expression at the DM and atrial $(A)$ dorsal wall is weaker corresponding with the expression pattern of alpha-smoothmuscle actin ( $\alpha \mathrm{SMA}, h)$, which marks the developing myocardium. In knockouts, a larger region shows less expression of MLC2a $(c, e), \operatorname{Nkx} 2.5(g)$ and $\alpha$ SMA $(i)$ corresponding with altered differentiation rate of myocardium at these regions. Left cardinal vein (LCV), Lung (L). Scale bars $30 \mu \mathrm{m}$.

Table 2). In wild types, the primitive PV myocardium in the region of the dorsal mesocardium had mosaic Nkx2.5 expression, defined as a mixture of $\mathrm{Nkx} 2.5$ positive and negative cells. Similar to MLC2a expression, the Nkx2.5 mosaic myocardial cells were mainly situated on the left side of the dorsal mesocardium (Fig. 1f). Moreover, in knockouts, this area was hypoplastic showing less $\mathrm{Nkx} 2.5$ expressing cells (compare Fig. $1 f$ with $g$ ).

The alpha-smooth-muscle actin antibody 1A4 was coexpressed in the MLC2a-stained myocardium of the common atrium and ventricle of the wild-type embryos. Similar to MLC2a, actin expression around the primitive PV and left part of the atrial dorsal wall was weaker (Fig. $1 h$ ). In mutants, actin expression was diminished in a larger region compared with the wild type (Fig. 1i).
E12.5. The common PV bifurcated into tiny left and right PVs, covered by a MLC2a-positive myocardial layer, which, in contrast to E10.5, could now be observed in equal density on both sides of the PV (Fig. 2a, c). MLC2a expression around the PV was weaker than the expression in the myocardium of the atria (Fig. 2a, c). In the dorsal mesocardium and around the PV, Nkx2.5 expression was mosaic, whereas in the wall of the cardinal veins $\mathrm{Nkx} 2.5$ staining was negative (Fig. $2 d$ ). In the atrial myocardium of the wild-type embryos, MLC2a and Nkx2.5 were positive, whereas smooth muscle actin had almost disappeared, particularly in the LA dorsal wall (Fig. 2e). In the myocardium of the ventricles (not shown) and in the wall of the cardinal veins (Fig. 2e), smooth muscle actin was still present. Actin expression in the wall of the PV was more extensive than the MLC2a expression (Fig. 2e).

In the podoplanin knockout embryos, MLC2a (Fig. $2 f, g$ ) and actin positive (Fig. $2 i$ ) as well as Nkx2.5 mosaic (Fig. 2h) myocardium around the PV was thin and locally even absent (compare Fig. 2a, $c$ with $f, g$ ) in a region where podoplanin expression was seen in wild-type embryos (Fig. 2b). The LA dorsal wall and the atrial septum were thin and the myocardium was hypoplastic (compare Fig. $2 j, k$ with $l, m$ ). The atrial septum showed a large secondary foramen and myocardialization at the base of the atrial septum was absent (Fig. 2k, $m$ ). Additionally, the atrioventricular cushion was not fused properly to the top of the ventricular septum resulting in an interventricular communication (Fig. $2 j, l$ ).

$\boldsymbol{E}$ 15.5. In both wild types and knockouts, the myocardium of the atria, the atrial septum and of the wall of the cardinal, and PVs expressed MLC2a (Fig. 3a-f). In mutants, the myocardium of the atrial septum and the PVs was hypoplastic (compare Fig. $3 c, d$ with $e, f$ ). Moreover, compared with wild type, the PVs in the knockout embryos seemed to be dilated (compare Fig. $3 c$ with $e$ ). In both wild-type and knockout embryos, the Nkx2.5 mosaic expression in the wall of the PV became overall positive (Fig. $3 g, i$ ).

In wild-type embryos, 1A4 costaining was specifically observed in the MLC2a positive subendothelial layer of the PVs and LA dorsal wall (Fig. 3h). In the outer MLC2a positive myocardial layer of the wall of the PVs, cardinal veins, dorsal atrial wall, and the entire atrial myocardium (not shown) 1A4 staining had disappeared (Fig. 3h). In knockout embryos, smooth muscle actin was almost absent in the subendothelial layer of the PVs and LA dorsal wall, which still showed MLC2a staining (compare Fig. $3 a, h$ with $b, j$ ). In areas with 1A4 expression, distribution of positive cells was discontinuous compared with the wild-type embryos (compare Fig. $3 h$ with $j$ ).

E18.5. The common part of the PVs was incorporated into the LA. In both wild-type and knockout embryos, MLC2a expressing myocardium was seen in the atria and the wall of the cardinal and PVs, whereas the myocardium now extended into the lungs (Fig. $4 a, b$ ). Similar to previous stages, the myocardium was hypoplastic in knockouts, with dilation of the atria, the cardinal, and PVs (compare Fig. $4 a, b$ with $c, d$ ). In the dorsal mesocardium, less mesenchymal cells were observed compared with the wild type (compare asterisk in Fig. $4 a$ with $b$ ). 


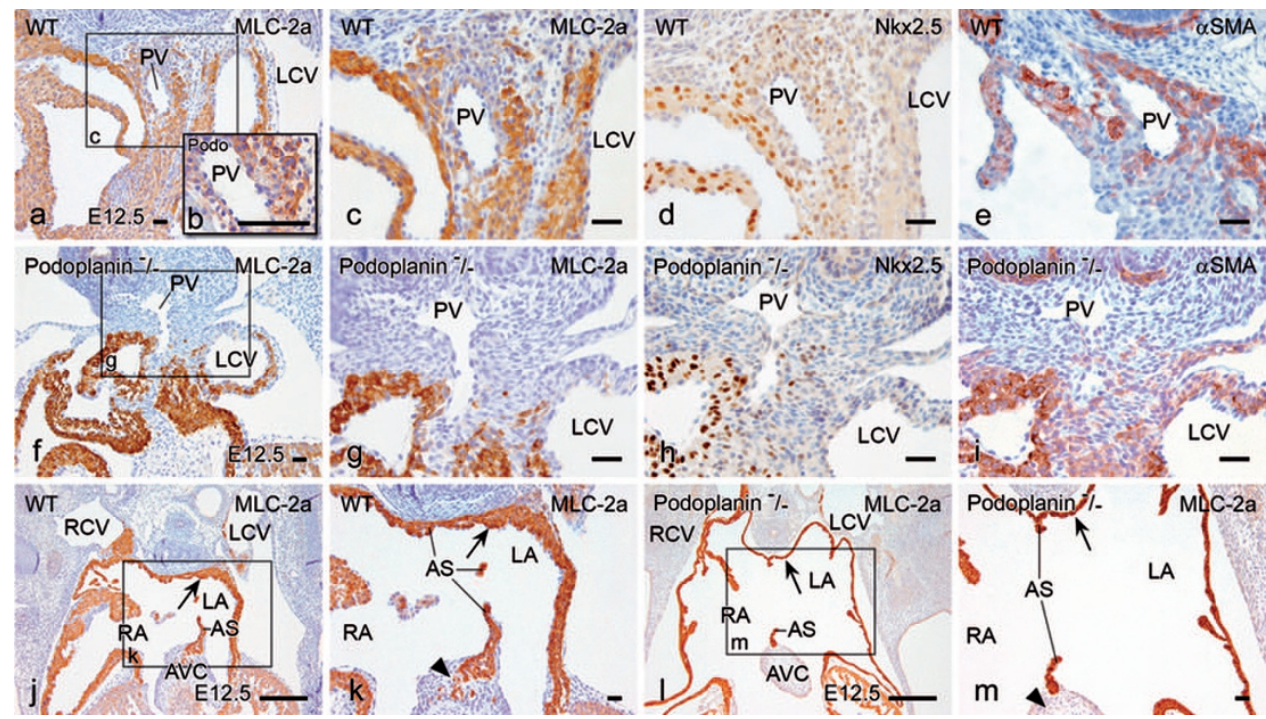

Figure 2. Transverse sections of podoplanin wild type (WT, $a-e, j, k$ ) and knockout (Podoplanin-l-, $f-i, l, m$ ) mouse embryos of stage (E) 12.5 comparing the development of the pulmonary vein (PV, $a-i)$, atrial septum (AS, $j-m$ ), and left atrial (LA) dorsal wall $(j-m)$. In WT embryos PV is surrounded by myocardium expressing MLC2a $(a, c)$, podoplanin $(b)$ and mosaic Nkx2.5 $(d)$ and completely positive for alpha-smooth-muscle actin $(\alpha \mathrm{SMA}, e)$. In mutants, the expression of these markers around the PV is almost absent (compare $a, c-e$ with $f-i$ ) and myocardium of the LA dorsal wall is hypoplastic (compare arrow in $j, k$ with $l, m)$. The AS in the knockouts is thin and deficient with a large secondary foramen $(k, m)$. Moreover, myocardialization process of the AS with the deficient atrioventricular cushion (AVC, compare $j$ with $l$ ) is absent (see arrowhead in $k$ and $m$ ), which might cause an atrioventricular septal defect. Boxed area in $a, f, j$ and $l$ are magnified in $c, g, k$, and $m$. Left cardinal vein (LCV), right atrium (RA), right cardinal vein (RCV). Scale bars $a-i, k, m 30 \mu \mathrm{m}, j, l 200 \mu \mathrm{m}$.

In the PV and LA dorsal wall of the wild-type embryos, smooth muscle actin (1A4) was present between endothelium and MLC2a positive myocardium (Fig. 4e). Hence, the 1A4 positive actin layer was MLC2a negative in contrast to the previous stages (compare Fig. $3 d$, $h$ with Fig. $4 c, e$ ). The 1A4 staining at other parts of the heart was absent (not shown), except for the wall of the cardinal veins and ventricular myocardium. Again, hardly any 1A4 staining was seen in the subendothelial layer of the PV and LA dorsal wall of the podoplanin knockout embryos (compare Fig. $4 e$ with $f$ ).

\section{DISCUSSION}

During human development, a vascular lining comprising SMCs and an adventitia will evolve at the inside of the LA body that is lined on the outside by atrial myocardium (14). Findings in the current study show a similar phenomenon in the LA of wild-type mouse embryos. A difference between mouse and human specimen concerns the extension of the myocardial tissue surrounding the PV into the lung. The merits of our current study are that the posterior heart field plays a role in the addition of not only PV myocardium, but also in the formation and differentiation of SMCs that line the LA body. We demonstrated hypoplasia of the myocardium of the PV, LA dorsal wall, and the atrial septum in podoplanin knockout mice. The vessel wall of the PVs and its extension into the LA was underdeveloped in knockout embryos.

Myocardial development. It has been reported earlier that the myocardial sleeve around the PV is formed by either migration of existing atrial cardiomyocytes (1) or by recruitment and differentiation of mesenchymal cells of the splanchnic mesoderm (2). The latter supports earlier advances in the study of cardiac development that underline the relevance of addition of myocardium to the primary heart tube (15). The second heart field (5) or second lineage $(5,4)$ concerns an anteroposterior extension of splanchnic mesenchyme from where cells are recruited for addition to both the arterial pole and the venous pole of the heart. Several studies have been performed using different lineage markers such as fibroblast growth factor (Fgf) 8 and 10 (16), Islet (Isl)I (5), and Tbxl and $18(17,18)$ to trace these cells into their cardiac destination. Special interest was raised in markers specific for recruitment of myocardium from the second heart field to the venous pole of the heart such as Pitx2c (3), Nkx2.5 (3,7), Shox2 (19), and podoplanin $(7,8)$.

Podoplanin promotes EMT by binding ERM proteins that activate RhoA and by down-regulation of the cell-to-cell adhesion molecule E-cadherin $(8,9)$. In podoplanin knockout mice, E-cadherin is up-regulated causing abnormal EMT, which may lead to abnormal formation of myocardium at the venous pole of the heart (8). Thus, the hypoplasia of the myocardium of the wall of the PVs, atrial septum, and LA dorsal wall observed in the current study in podoplanin knockout mice could be explained by impaired addition of myocardium from the posterior heart field because of abnormal EMT by lack of podoplanin.

Another explanation for the myocardial hypoplasia in the podoplanin knockouts could be abnormal epicardialmyocardial interaction. Previously, we demonstrated that altered epicardial-myocardial interaction leads to deficient ventricular myocardial formation in SP3 mutants (20) as well as in podoplanin mutant embryos (8). In SP3 mutants, WT-1 expression, a transcription factor involved in development of epicardium derived cells (EPDCs) (21) was down-regulated and the mutant hearts showed EPDC-related cardiac abnormalities comparable with podoplanin mutants. The impaired formation of EPDCs and altered epicardial-myocardial inter- 


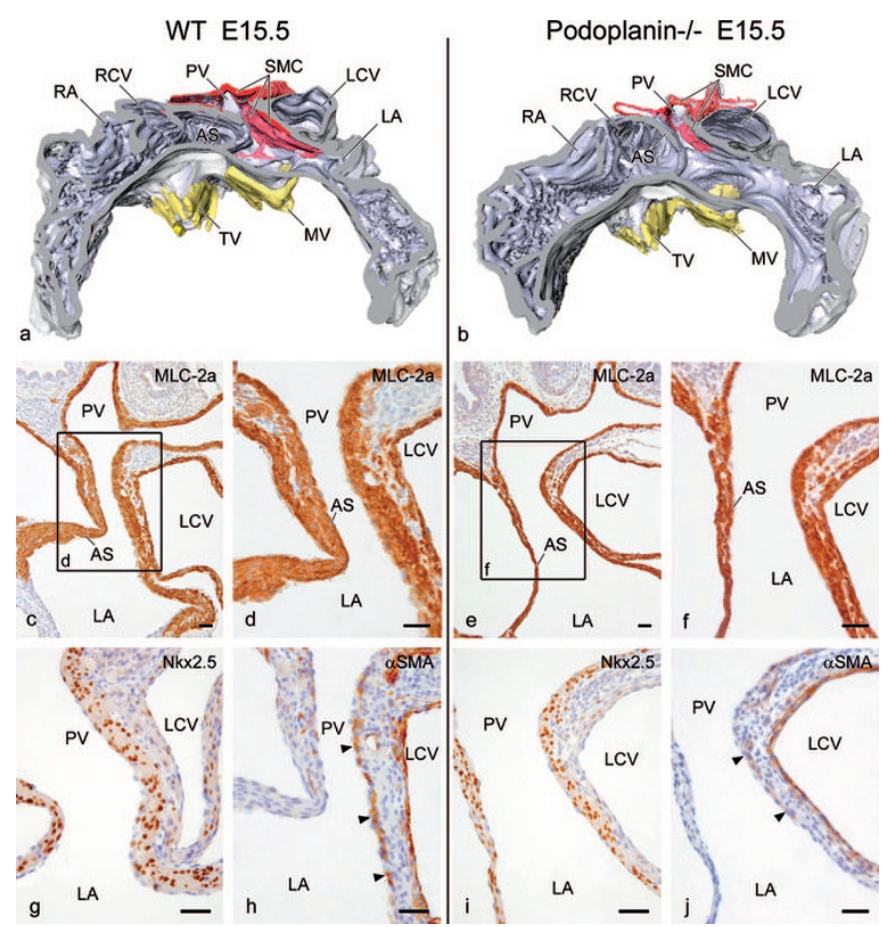

Figure 3. 3D reconstructions $(a, b)$ and transverse sections $(c-j)$ of podoplanin wild type (WT, $a, c, d, g, h$ ) and knockout (Podoplanin - $-, b, e, f$, $i, j)$ mouse embryos of stage (E) 15.5 showing hypoplasia of atrial septum (AS) and altered smooth muscle cells (SMC red) formation in the wall of the common pulmonary vein (PV) and the posterior wall of left atrium (LA) of the mutants. $a$ and $b$ are a cranial view of the PV orifice into the LA showing the diminished amount of SMCs in the PV and LA dorsal wall in knockouts. Boxes in $c$ and $e$ are the positions of the enlargements in $b$ and $f$. Compared with WT embryos, the MLC2a positive myocardium of the AS, the left cardinal vein (LCV), and the somewhat dilated PVs in knockouts is hypoplastic with interposition of less mesenchymal tissue (compare $c, d$ with $e, f$ ). The myocardium of the wall of the $\mathrm{PV}$ is differentiated and is seen as a myocardial cuff around the PV, which expresses Nkx2.5 $(g, i)$. In WT embryos, SMCs (arrowheads in $h$ ) are incorporated into the LA, whereas in knockouts SMCs are almost absent (arrowheads in $j$ ). Mitral valve (MV, yellow), Right atrium lumen (RA, light gray), Right cardinal vein lumen (RCV, light gray), tricuspid valve (TV, yellow). Color codes: light gray (lumen), dark gray (MLC2a positive myocardium). Scale bars $30 \mu \mathrm{m}$.

action, resulting in hypoplastic atrial and ventricular myocardium in podoplanin knockouts, has already been described and related to the deficient contribution of the posterior heart field $(8,22)$. Therefore, the hypoplasia of the PVs, atrial septum, and LA dorsal wall reported in the current study could be related to the altered contribution of epicardium and myocardium from the posterior heart field.

In early stages, Nkx2.5 and alpha-smooth-muscle actin (1A4) are expressed in undifferentiated myocardium. At these stages, Nkx2.5 is mosaic around the PVs and absent in the wall of the cardinal veins (7), whereas actin expression is present in the wall of the pulmonary and cardinal veins. The Nkx2.5 mosaic expression in the wall of the PVs rapidly becomes completely positive concomitant with a higher proliferation rate of the PV myocardium compared with the cardinal veins (3). Consequently, 1A4 expression from E15.5 is confined to the medial layer of the vascular wall of the pulmonary and cardinal veins. These findings suggest that the pulmonary and cardinal veins have a common precursor derived from the posterior heart field (7), but a distinct

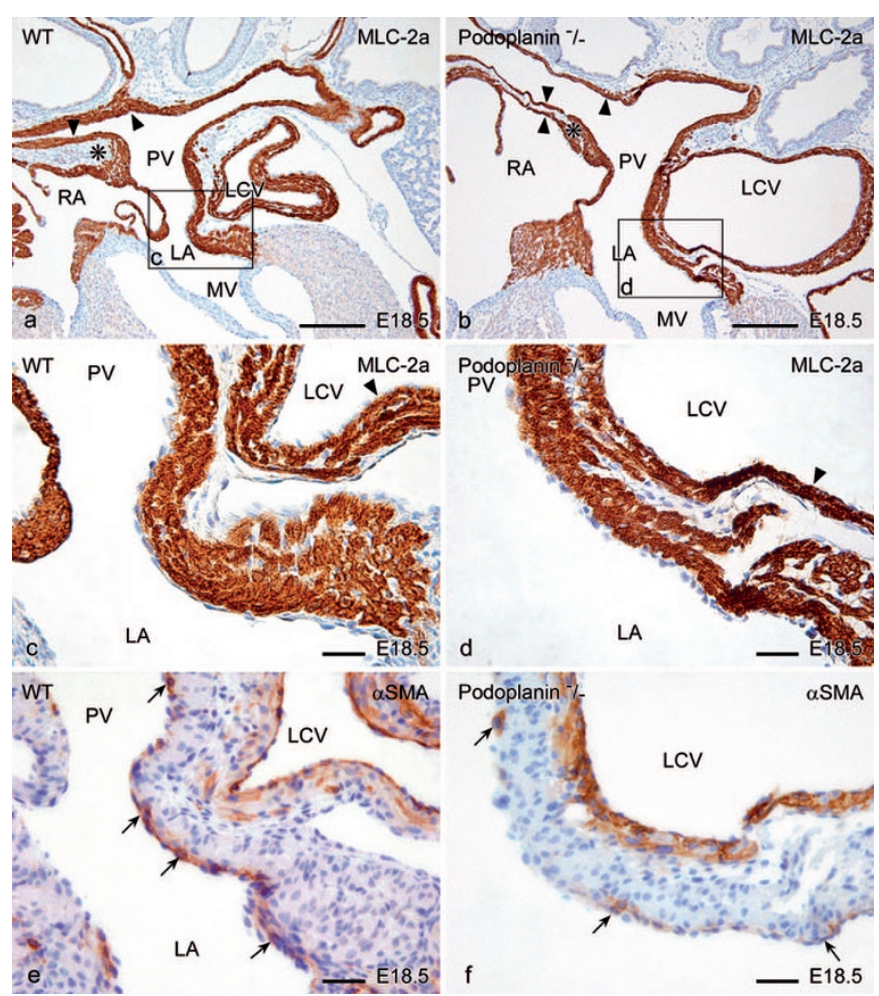

Figure 4. Transverse sections of podoplanin wild type (WT, $a, c, e$ ) and knockout (Podoplanin- $-, b, d, f$ ) mouse embryos of stage (E) 18.5 comparing the development of pulmonary vein (PV). Sections $a-d$ are stained with MLC2a as myocardial marker and sections $e$ and $f$ are stained with alpha-smooth-muscle cell actin ( $\alpha$ SMA). Boxes in $a, b$ are the positions of the enlargements in $c, d . a, b$. MLC2a expression is seen in WT and mutants in the myocardium of left (LA) and right atrium (RA), left cardinal vein (LCV) and pulmonary veins (PV) $(a, b)$. In knockouts, these structures are dilated and have hypoplastic myocardium (arrowheads in $a-d$ ) with interposition of less mesenchymal tissue ( $a, b$; asterisks). SMCs are seen in the subendothelial layer of the PV and LA dorsal wall (arrows in e). In the mutants, SMCs are almost absent, which might be caused by either impaired SMC formation or differentiation because of the lack of podoplanin (compare arrows in $e$ with $f$ ). Mitral valve (MV). Scale bars $a, b 200 \mu \mathrm{m} ; c-f 30 \mu \mathrm{m}$.

proliferation (3) rate accounting for a distinct differentiation based on $\mathrm{Nkx} 2.5$ expression.

Expression of markers in putative PV myocardium starts at the left side of the dorsal mesocardium, indicating that PV myocardium is preferentially added from the left side of the posterior heart field regulated by progenitor cells that play a role in left-right patterning, as was reported for Pitx $2 \mathrm{c}$ $(3,23,24)$. Next to hypoplasia, mutants showed diminished expression, which was predominantly observed in the earlier stages (Tables 1 and 2). As in later stages, these differences were no longer apparent, this suggests a delayed differentiation of the myocardium and SMCs in mutants.

Smooth muscle cell development. The origin of the SMCs at the venous pole is as yet not well understood. SMCs may differentiate from mesenchymal cells (6). At the arterial pole, formation and differentiation of SMCs has been reported from the splanchnic mesoderm or from the neural crest (25). The latter process requires cross-talk between the endothelial and the muscular component (26). DeRuiter et al. (27) have described the formation of the SMCs of the dorsal aorta by transdifferentiation from endothelial cells. 
Research in this field is complicated as alpha-smoothmuscle actin also stains the primitive myocardium. We demonstrated that this staining was less extensive in developing myocardium of the podoplanin mutant mice, supporting delayed or defective myocardial differentiation. At stage 15-18 in which alpha-smooth-muscle actin disappears from the myocardium, only the SMCs retain their expression of this marker. In podoplanin knockout mice, we observed a diminished extension of SMCs in the LA dorsal wall compared with normal. This phenomenon may be caused by impaired formation of SMCs from the posterior heart field-derived dorsal mesocardium or abnormal or delayed differentiation of the SMCs in the PV and LA body in absence of podoplanin. The disturbed EMT in podoplanin knockouts probably leads to abnormal formation of SMCs, as was shown for the coronary artery SMCs development in mutant mice (8).

In conclusion, as podoplanin is a marker of the myocardial and mesenchymal cells derived from the second heart field at the venous pole of the heart, this study supports evidence that the myocardium of the PVs, LA dorsal wall, and atrial septum is derived from the posterior heart field. Moreover, we can state that podoplanin not only plays a role in the development of myocardium, but also in the formation of the SMCs.

The clinical relevance of our findings needs further research. In the mutants, complex atrial defects were observed. In the majority, there was fusion of the primary atrial septum with the AV cushions, but the secondary foramen was enlarged and the AV cushions did not fuse properly with the ventricular septum resulting in an AVSD with shunting at the ventricular level. These findings were described in a previous study (8). The study of human and mouse models with isomerism, atrial arrhythmias, and cases with abnormal pulmonary venous return are on their way. More insight into the variation in myocardial cuffing of the PVs in the human population might enlighten us on the variability of occurrence of ectopic automaticity in the PV myocardial sleeve (28), which is suggested by the finding that the length of the PV sleeve corresponds to the frequency of occurrence of ectopic PV beats as observed in electrophysiological studies $(28,29)$. In podoplanin knockout embryos, we have observed deficient sinus venosus myocardium with myocardial discontinuities. Areas lacking myocardium can be regarded as low voltage areas (comparable with scar tissue) that may form the substrate of reentry circuits. Electrophysiological testing in mutant mice is necessary to further investigate this hypothesis.

Acknowledgments. We thank Bert Wisse for preparation of the 3D-reconstructions, and Jan Lens for preparation of the figures.

\section{REFERENCES}

1. Millino C, Sarinella F, Tiveron C, Villa A, Sartore S, Ausoni S 2000 Cardiac and smooth muscle cell contribution to the formation of the murine pulmonary veins. Dev Dyn 218:414-425

2. Kruithof BP, van den Hoff MJ, Tesink-Taekema S, Moorman AF 2003 Recruitment of intra and extracardiac cells into the myocardial lineage during mouse development. Anat Rec A Discov Mol Cell Evol Biol 271:303-314

3. Mommersteeg MT, Brown NA, Prall OW, de Gier-de VC, Harvey RP, Moorman AF, Christoffels VM 2007 Pitx2c and Nkx2-5 are required for the formation and identity of the pulmonary myocardium. Circ Res 101:902-909

4. Kelly RG 2005 Molecular inroads into the anterior heart field. Trends Cardiovasc Med 15:51-56
5. Cai CL, Liang X, Shi Y, Chu PH, Pfaff SL, Chen J, Evans S 2003 Isl1 identifies a cardiac progenitor population that proliferates prior to differentiation and contributes a majority of cells to the heart. Dev Cell 5:877-889

6. Sartore S, Lenzi M, Angelini A, Chiavegato A, Gasparotto L, De Coppi P, Bianco R, Gerosa G 2005 Amniotic mesenchymal cells autotransplanted in a porcine model of cardiac ischemia do not differentiate to cardiogenic phenotypes. Eur J Cardiothorac Surg 28:677-684

7. Gittenberger-de Groot AC, Mahtab EA, Hahurij ND, Wisse LJ, DeRuiter MC, Wijffels MC, Poelmann RE 2007 Nkx2.5 negative myocardium of the posterior heart field and its correlation with podoplanin expression in cells from the developing cardiac pacemaking and conduction system. Anat Rec (Hoboken) 290:115-122

8. Mahtab EA, Wijffels MC, van den Akker NM, Hahurij ND, Lie-Venema H, Wisse LJ, DeRuiter MC, Uhrin P, Zaujec J, Binder BR, Schalij MJ, Poelmann RE, Gittenberger-de Groot AC 2008 Cardiac malformations and myocardial abnormalities in podoplanin knockout mouse embryos: correlation with abnormal epicardial development. Dev Dyn 237:847-857

9. Martin-Villar E, Megias D, Castel S, Yurrita MM, Vilaro S, Quintanilla M 2006 Podoplanin binds ERM proteins to activate RhoA and promote epithelialmesenchymal transition. J Cell Sci 119:4541-4553

10. Wetterwald A, Hoffstetter W, Cecchini MG, Lanske B, Wagner C, Fleisch H, Atkinson M 1996 Characterization and cloning of the E11 antigen, a marker expressed by rat osteoblasts and osteocytes. Bone 18:125-132

11. Williams MC, Cao Y, Hinds A, Rishi AK, Wetterwald A 1996 T1 alpha protein is developmentally regulated and expressed by alveolar type I cells, choroid plexus, and ciliary epithelia of adult rats. Am J Respir Cell Mol Biol 14:577-585

12. Breiteneder-Geleff S, Matsui K, Soleiman A, Meraner P, Poczewski H, Kalt R, Schaffner G, Kerjaschki D 1997 Podoplanin, novel 43-kd membrane protein of glomerular epithelial cells, is down-regulated in puromycin nephrosis. Am J Pathol 151:1141-1152

13. Schacht V, Ramirez MI, Hong YK, Hirakawa S, Feng D, Harvey N, Williams M, Dvorak AM, Dvorak HF, Oliver G, Detmar M 2003 T1alpha/podoplanin deficiency disrupts normal lymphatic vasculature formation and causes lymphedema. EMBO J 22:3546-3556

14. Douglas YL, Jongbloed MR, Gittenberger-de Groot AC, Evers D, Dion RA, Voigt P, Bartelings MM, Schalij MJ, Ebels T, DeRuiter MC 2006 Histology of vascular myocardial wall of left atrial body after pulmonary venous incorporation. Am J Cardiol 97:662-670

15. De la Cruz MV, Castillo MM, Villavicencio L, Valencia A, Moreno-Rodriguez RA 1997 Primitive interventricular septum, its primordium, and its contribution in the definitive interventricular septum: in vivo labelling study in the chick embryo heart. Anat Rec 247:512-520

16. Kelly RG, Brown NA, Buckingham ME 2001 The arterial pole of the mouse heart forms from Fgf10-expressing cells in pharyngeal mesoderm. Dev Cell 1:435-440

17. Xu H, Morishima M, Wylie JN, Schwartz RJ, Bruneau BG, Lindsay EA, Baldini A $2004 \mathrm{Tbx} 1$ has a dual role in the morphogenesis of the cardiac outflow tract. Development 131:3217-3227

18. Christoffels VM, Mommersteeg MT, Trowe MO, Prall OW, Gier-de Vries C, Soufan AT, Bussen M, Schuster-Gossler K, Harvey RP, Moorman AF, Kispert A 2006 Formation of the venous pole of the heart from an Nkx2-5-negative precursor population requires Tbx18. Circ Res 98:1555-1563

19. Blaschke RJ, Hahurij ND, Kuijper S, Just S, Wisse LJ, Deissler K, Maxelon T, Anastassiadis K, Spitzer J, Hardt SE, Schöler H, Feitsma H, Rottbauer W, Blum M, Meijlink F, Rappold GA, Gittenberger-de Groot AC 2007 Targeted mutation reveals essential functions of the homeodomain transcription factor Shox 2 in sinoatrial and pacemaking development. Circulation 115:1830-1838

20. Van Loo PF, Mahtab EA, Wisse LJ, Hou J, Grosveld F, Suske G, Philipsen S, Gittenberger-de Groot AC 2007 Transcription Factor Sp3 knockout mice display serious cardiac malformations. Mol Cell Biol 27:8571-8582

21. Moore AW, McInnes L, Kreidberg J, Hastie ND, Schedl A 1999 YAC complementation shows a requirement for $\mathrm{Wt1}$ in the development of epicardium, adrenal gland and throughout nephrogenesis. Development 126:1845-1857

22. Lie-Venema H, Eralp I, Markwald RR, Van Den Akker NM, Wijffels M, Kolditz DP, Van der Laarse A, Schalij MJ, Poelmann RE, Bogers A, Gittenberger-de Groot AC 2008 Periostin expression by epicardium-derived cells (EPDCs) is involved in the development of the atrioventricular valves and fibrous heart skeleton. Differentiation 76:809819

23. Franco D, Campione M 2003 The role of Pitx 2 during cardiac development. Linking left-right signaling and congenital heart diseases. Trends Cardiovasc Med 13:157-163

24. Poelmann RE, Jongbloed MR, Gittenberger-de Groot AC 2008 Pitx2: a challenging teenager. Circ Res 102:749-751

25. Rosenquist TH, Beall AC 1990 Elastogenic cells in the developing cardiovascular system. Smooth muscle, nonmuscle, and cardiac neural crest. Ann N Y Acad Sci 588:106-119

26. Wang HU, Chen ZF, Anderson DJ 1998 Molecular distinction and angiogenic interaction between embryonic arteries and veins revealed by ephrin-B2 and its receptor Eph-B4. Cell 93:741-753

27. DeRuiter MC, Poelmann RE, VanMunsteren JC, Mironov V, Markwald RR, Gittenberger-de Groot AC 1997 Embryonic endothelial cells transdifferentiate into mesenchymal cells expressing smooth muscle actins in vivo and in vitro. Circ Res 80:444-451

28. Haissaguerre M, Jais P, Shah DC, Takahashi A, Hocini M, Quiniou G, Garrigue S, Le MA, Le MP, Clementy J 1998 Spontaneous initiation of atrial fibrillation by ectopic beats originating in the pulmonary veins. N Engl J Med 339:659-666

29. Ho SY, Sanchez-Quintana D, Cabrera JA, Anderson RH 1999 Anatomy of the left atrium: implications for radiofrequency ablation of atrial fibrillation. J Cardiovasc Electrophysiol 10:1525-1533 